\title{
Febrile urinary tract infection in children: changes in epidemiology, etiology, and antibiotic resistance patterns over a decade
}

Woosuck Suh, MD, Bi Na Kim, MD, Hyun Mi Kang, MD, Eun Ae Yang, MD, Jung-Woo Rhim, MD, Kyung-Yil Lee, MD

Departments of Pediatrics, College of Medicine, The Catholic University of Korea, Seoul, Korea

Background: Understanding the epidemiology and prevalence of febrile urinary tract infection (fUTI) in children is important for risk stratification and selecting appropriate urine sample collection candidates to aid in its diagnosis and treatment.

Purpose: This study aimed to analyze the epidemiology, etiology, and changes in antibiotic susceptibility patterns of the first fUTI in children.

Methods: This retrospective observational cohort study included children younger than 19 years of age who were diagnosed and treated for their first fUTI in 2006-2016. Electronic medical records were analyzed and radiologic images were evaluated.

Results: A total of 359 patients (median age, 5.1 months; interquartile range, $3.0-10.5$ months) fit the inclusion criteria; of them, $78.0 \%(n=280)$ were younger than 12 months old. The male to female ratio was 5.3:1 for patients aged 0-2 months, 2.1:1 for those 3-5 months, and 1.6:1 for those 6-11 months. Beyond 12 months of age, there was a female predominance. Escherichia coli was the leading cause (83.8\%), followed by Enterococcus species (6.7\%), and Klebsiella pneumoniae $(3.6 \%)$. Significant yearly increases in the proportions of multidrug-resistant strains $(P<0.001)$ and extended-spectrum beta-lactamase $(\mathrm{ESBL})$ producers $(P<0.001)$ were observed. In patients with vesicoureteral reflux (VUR), the overall recurrence rate was $53.6 \%(\mathrm{n}=15)$. A significantly higher recurrence rate was observed when the fUTI was caused by an ESBL versus non-ESBL producer $(75.0 \%$ vs. $30.0 \%, P=0.03)$.

Conclusion: fUTI was most prevalent in children younger than 12 months of age and showed a female predominance in patients older than 12 months of age. The proportion of ESBL producers causing fUTI is increasing. Carbapenems, rather than noncarbapenems, should be considered for treating fUTI caused by ESBL-producing enteric gram-negative rods to reduce short-term recurrence rates in children with VUR.
Key words: Acute pyelonephritis, Etiology, Antibiotics susceptibility, Resistance

\section{Key message}

Question: How has the antibiotic susceptibility of urinary pathogens changed and what does it imply?

Finding: A yearly increase in multidrug-resistant and extendedspectrum $\beta$-lactamase (ESBL)-producing pathogens was observed. A higher recurrence rate was observed in cases of febrile urinary tract infection caused by ESBL producers in patients with underlying vesicoureteral reflux (VUR).

Meaning: The initial empirical antibiotic should reflect the changing susceptibility patterns and underlying VUR status.

\section{Introduction}

Febrile UTI (fUTI) remains as one of the most common serious bacterial infections in children; with approximately $8 \%$ of girls, and $2 \%$ of boys being affected before 8 years old. ${ }^{1,2)}$ With a high prevalence during the first year of life, ${ }^{3)}$ fUTI is a disease with usually a benign course in children without underlying renal pathology, provided that prompt diagnosis and treatment is given to prevent subsequent renal scaring. ${ }^{4,5)}$

Although numerous studies have been carried out on children, fUTI remains a diagnostic and therapeutic challenge to clinicians. Because fUTI is prevalent in infants below 24 months old who do not yet have verbal skills, ${ }^{3)}$ the presenting symptoms are nonspecific, and unexplained fever with irritability is usually the only complaint. Diagnosis of fUTI involves urine sample collection via invasive procedures such as suprapubic aspiration and bladder catheterization ${ }^{6}$ or time-consuming method such as sterile bag collection in children who are non-toilet-trained.") Therefore, clinicians are often faced with the challenge of selecting higher risk children who would benefit from the diagnostic testing. ${ }^{6,8)}$

\footnotetext{
Corresponding author: Hyun Mi Kang, MD. Department of Pediatrics, Seoul St. Mary's Hospital, College of Medicine, The Catholic University of Korea, 222 Banpo-daero, Seocho-gu, Seoul 06591, Korea 
Gut colonizing Enterobacteriaceae such as Escherichia coli, Klebsiella species, and Enterobacter species, are common causes of fUTI in children, and $\beta$-lactam antibiotics are recommended as first-line agents. Because antibiotic resistance has become a worldwide issue, and due to the increase in multidrug-resistant (MDR) Enterobacteriaceae, extended-spectrum $\beta$-lactams are now considered as first-line agents for empirical antibiotic therapy. Initiating treatment with oral antibiotics has shown to be as effective as parenteral antibiotics for the first episode of fUTI in children.9) However, recently, many countries have been reporting an increase in extended-spectrum $\beta$-lactamase (ESBL)producing Enterobacteriaceae; and thus, clinicians are now facing a dilemma in selecting empirical antibiotics for fUTI in children.

Understanding the epidemiology and prevalence of fUTI in children is important for risk stratification and selecting appropriate candidates for urine sample collection to aid in the diagnosis fUTI. Therefore, the primary aim of this study was to investigate whether any change has occurred in the epidemiology of fUTI in children. Furthermore, because empirical antibiotic choice relies on resistance patterns in a given region, the secondary aim of this study was to explore antibiotic resistance patterns of uropathogens in Daejon, South Korea.

\section{Methods}

\section{Study population}

This was a retrospective cohort study of patients below 19 years of age, who were diagnosed and treated for the first episode of fUTI between January 2006 and December 2016 at a secondary care referral university hospital located in Daejon, South Korea. A single medical team followed the same protocol for evaluation and treatment of all patients included in this study.

Only patients who fulfilled all of the following criteria were included in study: (1) fever within the previous 24 hours (body temperature $>38.0^{\circ} \mathrm{C}$ ), (2) pyuria (positive leukocytes of $\geq 10$ white blood cells per high-power field on urine microscopy), (3) positive urine culture, (4) first episode of fUTI, and (5) no underlying chronic disease such as neuromuscular, gastrointestinal, hemato-oncologic, or pulmonary diseases. ${ }^{8)}$

\section{Study protocol and data collection}

Patients admitted for fever and pyuria were initiated with parenteral cefotaxime and amikacin after urine samples for culture were collected on the day of admission. On the 3rd day of parenteral antibiotics, a follow-up urinalysis and urine culture were taken. When the initial urine culture and antibiotic susceptibility results were reported, usually after the 3rd day of culture, and an ESBL producer was cultured and was susceptible to amikacin, both antibiotics were maintained. If the pathogen was resistant to amikacin, however defervescence occurred and follow-up urinalysis showed improvement in pyuria, treatment was maintained. In cases where there was lack of clinical impro- vement, the antibiotic was switched to a carbapenem. The total duration of antibiotics, including oral antibiotics, was $\geq 10$ days.

The electronic medical records of patients included in this study were reviewed for the following information: birthdate, gender, admission and discharge date, duration of fever, and initial laboratory and urine culture results. Data on the following imaging studies were investigated: dimercaptosuccinic acid (DMSA) scan, kidney and bladder ultrasound, and voiding cystourethrogram (VCUG). All patients received DMSA scan, kidney and bladder ultrasound. If the findings were suspicious for VUR, VCUG was performed.

\section{Microbiology and antibiotic susceptibility}

Positive urine cultures underwent species identification and antibiotic susceptibility tests via MicroScan NegCombo Panel 32 (2006-2008) and Neg Breakpoint Combo Panel 44 (20092016) (Siemens Healthcare Diagnostics Inc., West Sacramento, CA, USA). The minimum inhibitory concentration was reviewed for all microorganisms and the cutoff for antibiotic susceptibility was determined using the Clinical and Laboratory Standards Institute (CLSI) guideline. ${ }^{10)}$ The presence of ESBL-producing microorganisms was confirmed by double-disc synergy test in accordance with the CLSI guideline.

\section{Definitions}

If urine was obtained by catheterization or voiding, $\geq 10^{5}$ colony-forming unit $(\mathrm{CFU}) / \mathrm{mL}$ of a single organism was considered positive, while $>10^{5} \mathrm{CFU} / \mathrm{mL}$ obtained via sterile bag collection was considered significant.

Enteric gram-negative rods (eGNRs) included the following: Citrobacter freundii, E. coli, Enterobacter species, Klebsiella species, Morganella morganii, Proteus mirabilis, and Serratia fonticola. Strains nonsusceptible to $\geq 1$ antibiotic in $\geq 3$ antimicrobial categories were defined as a MDR bacteria, in accordance with the definitions for acquired resistance as proposed by the European Centre for Disease Prevention and Control and Centers for Disease Control and Prevention. ${ }^{11)}$

Clinical improvement was defined as patients fulfilling all the following: no fever within 24 hours, follow-up urinalysis of improved pyuria, and negative conversion of urine culture. Adequate treatment was defined as clinical improvement regardless of the type antibiotics administered.

\section{Statistical methods}

All statistical analyses were performed with $\mathrm{R}$ ver. 3.2.1. ( $\mathrm{R}$ Foundation for statistical computing, Vienna, Austria). Categorical variables were analyzed using Fisher exact test, and continuous variable was analyzed using Mann-Whitney $U$ test. The chi-square test for Trend in Proportions was used to investigate the trends for MDR and ESBL-producing strains and antibiotics resistance. All the statistical tests performed were 2 -tailed and a $P$ value of $\leq 0.05$ was considered significant. 


\section{Results}

\section{Demographics and clinical characteristics}

During the 11-year study period between January 2006 and December 2016, a total of 821 patients below 19 years old were treated for fever and pyuria at the Catholic University of Korea, Daejon St. Mary's Hospital. After the inclusion criteria were applied, 462 patients were excluded and a total of 359 patients diagnosed with the first episode of culture-positive fUTI were included in the study. Of these, $58.8 \%(\mathrm{n}=211)$ were male, and the median age was 5.1 (interquartile range [IQR], 3.0-10.5) months old (Table 1).

Patients were admitted at a median of 2 (IQR, 1-3) days after fever onset, and the median fever duration was 3 (IQR, 2-5) days. Of the patients that underwent imaging studies, a total $45.5 \%$ ( $n=163$ of 358 ) had abnormal findings on kidney and bladder ultrasounds, such as pelviectasia/calyectasia, pelvicalyceal dilatation, ureter dilatation, or ill-defined increased or decreased echogenic areas. Abnormal DMSA scans were found in $67.0 \%$ ( $n=233$ of 348$)$ of the patients. Patients who had kidney ultrasound findings suggesting vesicoureteral reflux (VUR) underwent VCUG, and $8.9 \%(n=31$ of 359$)$ of the patients were found to have VUR (Table 1). Groups with normal and abnormal DMSA scans showed no significant difference in age, sex, pathogen, fever duration, VUR rate, or recurrence (all $P>0.05)$.

\section{Epidemiology and etiology}

Of the 359 patients diagnosed with fUTI, $56.5 \%(n=203)$ of the patients were below 6 months old, $78.0 \%(n=280)$ below

Table 1. Clinical characteristics of children diagnosed with febrile urinary tract infection $(\mathrm{N}=359)$

\begin{tabular}{lc}
\hline Characteristic & Value \\
\hline Male sex & $211(58.8)$ \\
Age (mo) & $5.1(3.0-10.5)$ \\
Total & $4.1(2.6-6.2)$ \\
Male & $8.0(4.6-44.0)$ \\
Female & $329(91.6)$ \\
Gram-negative Enterobacteriaceae ${ }^{a)}$ & $143(43.5)$ \\
MDR strain & $54(16.4)$ \\
ESBL producer & $2(1-3)$ \\
Fever day at admission (day) & $3(2-5)$ \\
Fever duration (day) & $6(5-7)$ \\
Duration of hospitalization (day) & $31(8.6)$ \\
Vesicoureteral reflux & $163(45.5)$ \\
Abnormal kidney ultrasonography) & $233(67.0)$ \\
Abnormal DMSA scanc) & $30(8.4)$ \\
\hline Recurrence &
\end{tabular}

Values are presented as number (\%) or median (interquartile range). DMSA, dimercaptosuccinic acid; ESBL, extended-spectrum beta-lactamase; MDR, multidrug resistance.

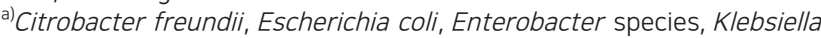
species, Morganella morganii, Proteus mirabilis, and Serratia fonticola.

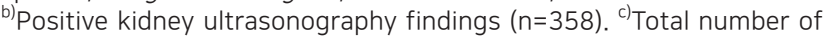
patients who underwent DMSA scans $(n=348)$.
12 months old, and $83.6 \%(n=300)$ below 24 months old (Fig. 1A). The median age of males diagnosed with fUTI was lower than females; 4.1 months (IQR, 2.6-6.2) versus 8.0 months (IQR, 4.6-44.0), respectively (Table 1). Male predominance was seen below 12 months old, with the male to female ratio 5.3:1 between ages 0 to 2 months, 2.1:1 between 3 to 5 months; and 1.6:1 between 6 to 11 months old. From 12 months and above, there was female predominance; with the female to male ratio above 1.9:1 up to 4 years old and beyond 17:1 from 5 years old (Fig. 1B).

E. coli was by far the most common cause of fUTI (83.8\%), followed by Enterococcus species (6.7\%), K. pneumoniae (3.6\%), and Enterobacter species (1.4\%) (Table 2).

\section{Antibiotic susceptibility}

Among eGNRs isolated during 2006 to $2010(n=100$ of 329) compared to 2011 to 2016 ( $n=229$ of 329), the extremely low antibiotic susceptibility rate to ampicillin and ampicillin-sulbactam decreased even further, from $34 \%$ to $26 \%$ and $42 \%$ to $32 \%$, respectively. The susceptibility rates to all generations of cephalosporin also decreased greater than $10 \%$ during the latter period. Amikacin and imipenem susceptibility rates remained high during the 2 periods, above 99\%; although during 2016, 2 imipenem resistant, meropenem sensitive, noncarbapenemase producing eGNRs were isolated. The $94 \%$ susceptibility rate to piperacillin-tazobactam did not change between the 2 periods. Ciprofloxacin, which had a higher susceptibility rate than pi-
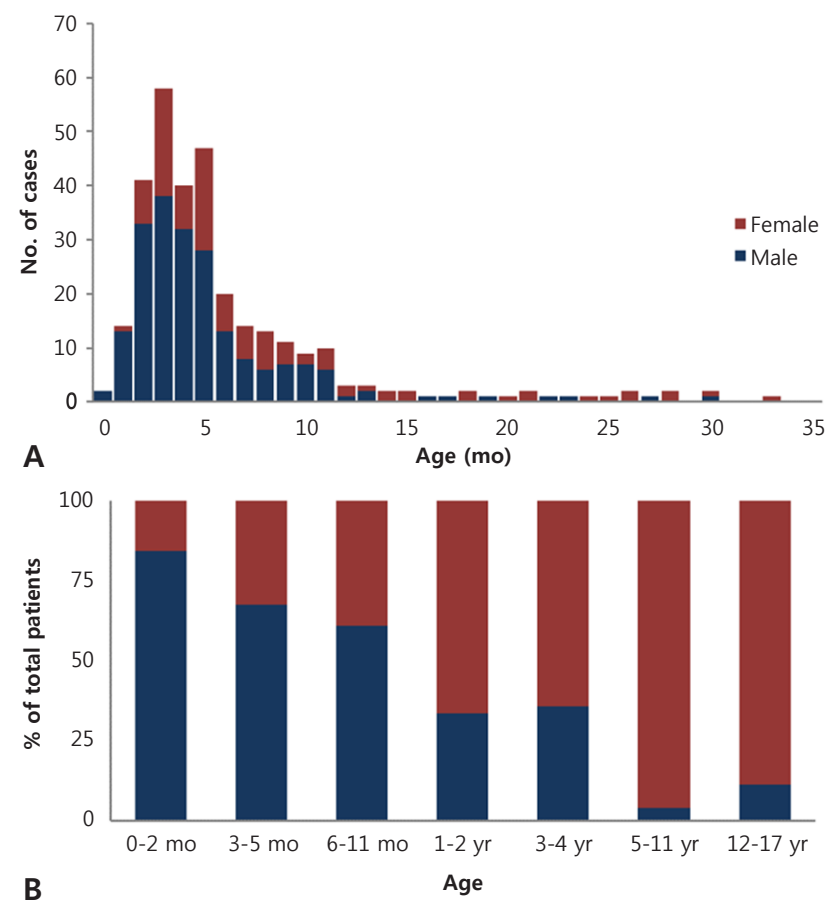

Fig. 1. Age and sex distribution. (A) Age distribution of the patients diagnosed with acute pyelonephritis at younger than 36 months of age showing that $78.0 \%$ ( $n=280$ of 359) were younger than 12 months old and $83.6 \%$ ( $n=300$ of 359) were younger than 24 months old. (B) Sex distribution of patients by age showing that a male predominance is seen only among patients younger than 12 months of age. 
peracillin-tazobactam during 2006-2010 decreased significantly (95\% to $87 \%$ ) during 2011-2016. The low susceptibility rate to trimethoprim-sulfamethoxazole during 2006 to 2010 remained similar during 2011 to 2016 (70\% vs. 68\%) (Fig. 2).

\section{Multidrug resistance and $\beta$-lactamase producing enteric gram-negative rods}

During the 11-year study period, a significantly increasing trend of MDR eGNRs causing fUTI was observed. In 2006, the proportion of MDR eGNRs causing fUTI was 35.7\% $(\mathrm{n}=5$ of 14), which increased to $51.9 \%(\mathrm{n}=28$ of 54$)$ in 2016 $(P<0.001)$. The same trend was observed with the proportion of fUTI caused by ESBL producers; 7.1\% ( $n=1$ of 14) in 2006 steadily increasing to $20.4 \%$ ( $n=11$ of 54$)$ in $2016(P<0.001)$ (Fig. 3). Fig. 4 shows the increase in resistance of eGNRs to each antibiotic. A significant linear increasing trend was observed for ampicillin-sulbactam, all generations of cephalosporin, ciprofloxacin, and imipenem.

Table 2. Etiology of acute pyelonephritis $(\mathrm{N}=359)$

\begin{tabular}{lc}
\hline Etiology & No. of cases (\%) \\
\hline Escherichia coli & $301(83.8)$ \\
Enterococcus species & $24(6.7)$ \\
Klebsiella pneumoniae & $13(3.6)$ \\
Enterobacter species & $5(1.4)$ \\
Klebsiella oxytoca & $4(1.1)$ \\
Staphylococcus species & $3(0.8)$ \\
Citrobacter freundii & $2(0.6)$ \\
Pseudomonas aeruginosa & $1(0.3)$ \\
Proteus mirabilis & $2(0.6)$ \\
Serratia fonticola & $1(0.3)$ \\
Streptococcus agalactiae & $1(0.3)$ \\
Candida glabrata & $1(0.3)$ \\
Morganella morganii & $1(0.3)$ \\
\hline
\end{tabular}

The clinical characteristics of fUTI caused by MDR versus non-MDR and ESBL producers versus non-ESBL producers were compared (Table 3). Although the median duration of hospitalization in both fUTI caused by MDR versus non-MDR $(P=0.012)$ and ESBL producers versus non-ESBL producers $(P=0.015)$ was statistically significant, the difference was less than one day in both groups. All other clinical characteristics depicting the severity of the disease, such as total fever duration, time to defervescence, leukocytosis, and C-reactive protein, showed no significant difference.

The recurrence rate of fUTI caused by eGNRs was investigated. In patients without VUR, the overall recurrence rate of fUTI was $4.9 \%(n=15$ of 301$) ; 6.5 \%(n=3$ of 46$)$ in fUTI caused by an ESBL producer versus $4.7 \%(n=12$ of 255$)$ in a non-ESBL producer $(P=0.602)$. All recurrences occurred 6 months after the initial fUTI diagnosis. However, in patients with any grade VUR, the overall recurrence rate was $53.6 \%(n=15$ of 28$)$; $75.0 \%$ ( $\mathrm{n}=6$ of 8 ) in fUTI caused by ESBL producers versus $45.0 \%(n=9$ of 20$)$ in non-ESBL producers $(P=0.150)$. The short-term recurrence rate within 6 months was determined in this patient group; a significantly higher recurrence rate was observed in cases where fUTI was caused by an ESBL producers versus non-ESBL producer ( $75.0 \%$ vs. $30.0 \%, P=0.03$ ) (Table $4)$.

\section{Discussion}

This was a study of 359 patients below 19 years old diagnosed with the first episode of fUTI during 2006 to 2016 . This study sought to observe whether any change in epidemiology has occurred in fUTI, and investigate antibiotic resistance patterns of urophathogens in Daeejon, South Korea, and its clinical implications.

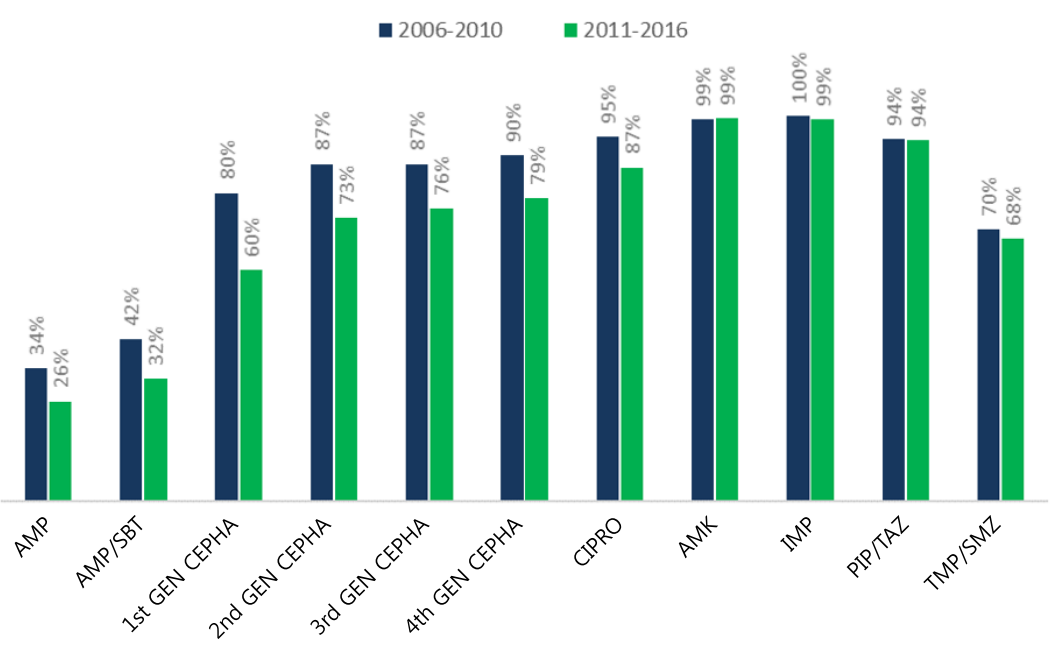

Fig. 2. Comparison of antibiotic susceptibilities between 2006-2010 and 2011-2016. Ampicillin (AMP), ampicillin-sulbactam (AMP/SULB), cefazolin (1st GEN CEPHA), cefuroxime (2nd GEN CEPHA), cefotaxime (3rd GEN CEPHA), cefepime (4th GEN CEPHA), ciprofloxacin (CIPRO)amikacin (AMK), piperacillin-tazobactam (PIP/TAZ), trimethoprim-sulfamethoxazole (TMP/SMZ). 
In this study, 2 main epidemiologic patterns were observed. First, the peak prevalence of fUTI was observed below 6 months of age, with over $50 \%$ of the entire study population in this age group. The overall median age of fUTI was 5.1 months old, and

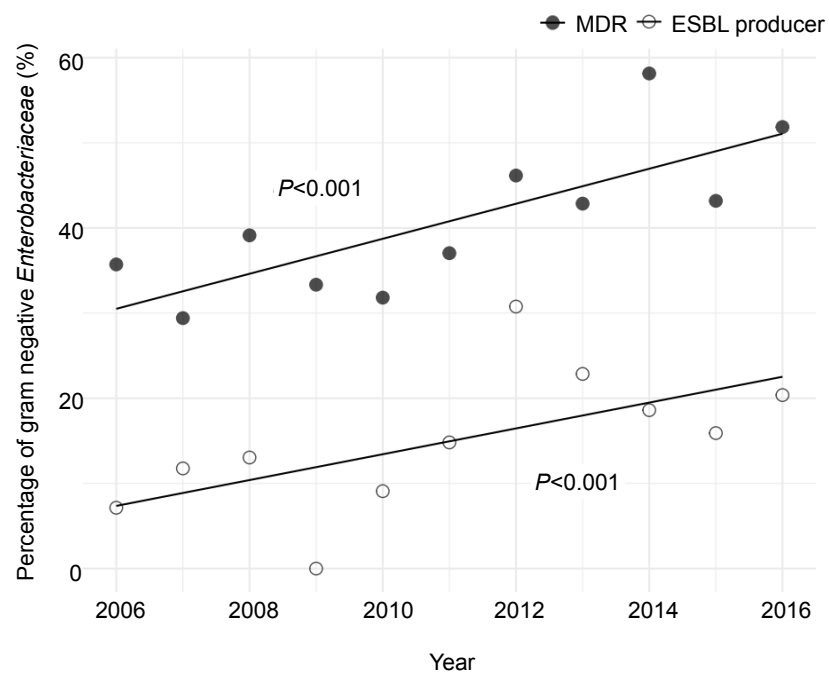

Fig. 3. Trend in proportion of MDR and ESBL-producing pathogens in fUTI. A significantly increasing trend of multidrug-resistant eGNRs causing fUTI was observed. The proportion of MDR eGNRs causing fUTI was $35.7 \%$ in 2006 versus $51.9 \%$ in $2016(P<0.001)$. The same trend was observed with the proportion of fUTI caused by ESBL producers: $7.1 \%$ in 2006 and $20.4 \%$ in $2016(P<0.001)$. eGNR, enteric gramnegative rods; ESBL, extended-spectrum beta-lactamase; fUTI, febrile urinary tract infection; MDR, multidrug-resistant. male predominance was seen below 1 years old. Beyond that, an extremely skewed distribution with female predilection of the disease was observed. A meta-analysis including patients diagnosed with UTI during 1966 to 2005 found similar epidemiologic patterns, with prevalence rates reported to be highest during the first 3 months of life and declining thereafter. And even among females, the prevalence rates were highest during the first 12 months of life, and beyond infancy, females had a higher risk of fUTI. ${ }^{3)}$ The reasons behind these distinct epidemiologic patterns of fUTI are unknown. However, many studies have attempted to explain these patterns on the structural differences in the genital areas influencing the variable risks of ascending infections, the immature immune status of children during infancy, ${ }^{12)}$ or the difference in the degree of periurethral/perineal colonization between the sexes which causes not only ascending infections but gut microbiota's bacterial translocation. ${ }^{13)}$

The burden of rapidly growing antibiotic resistance is an important issue recognized globally. Therefore, understanding the changes in antimicrobial susceptibility patterns in a given region is crucial for regulating the use of antibiotics, recognizing and controlling antimicrobial resistance, and effectively treating bacterial infections in both immunocompetent and immunocompromised patients. The extremely low antibiotic susceptibility rates of eGNRs to penicillins and penicillins plus $\beta$-lactamase inhibitors have already been recognized throughout the world. ${ }^{14,15)}$ In this study, during the 11 -year period, the low susceptibility rate was shown to decrease even further, nearing

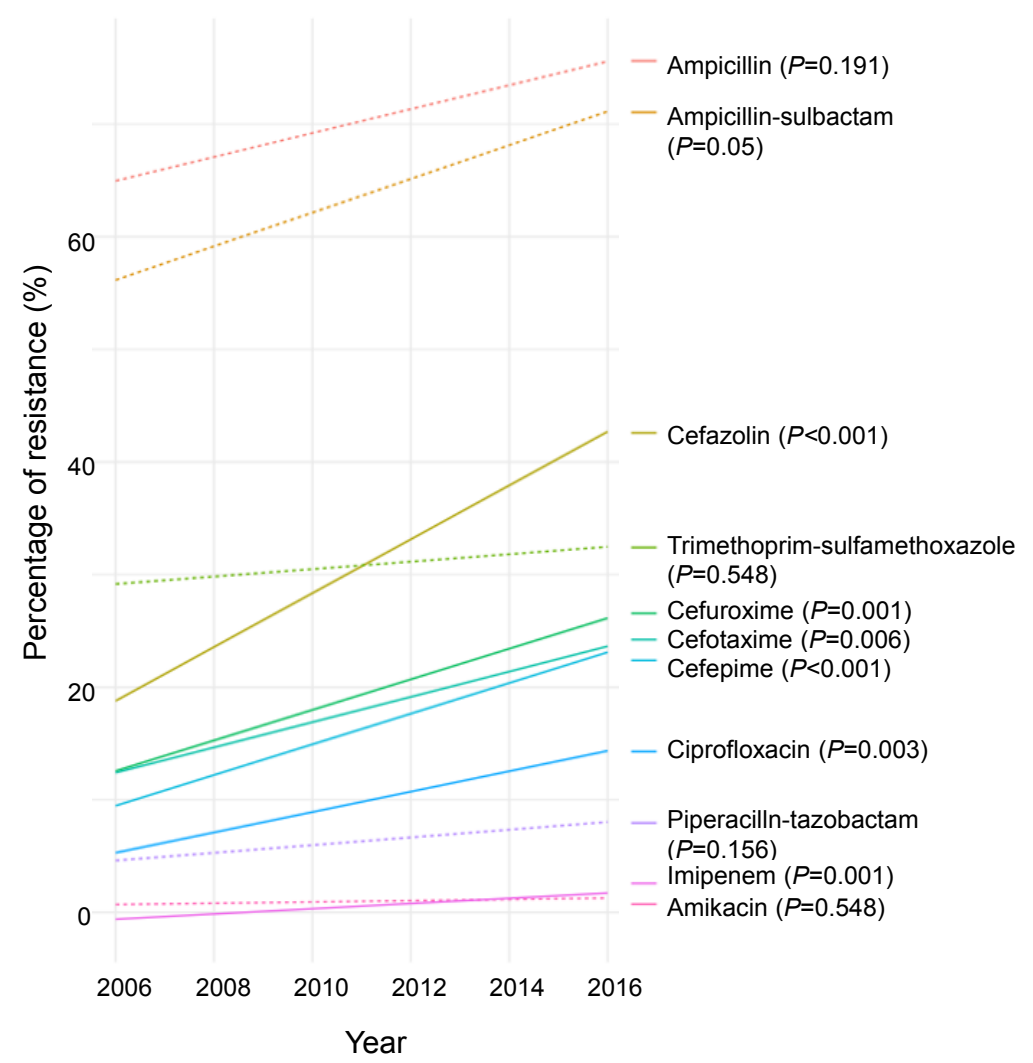

Fig. 4. Trend in the increase in eGNR resistance by antibiotic. A significant linear increasing trend was observed for ampicillin-sulbactam, all generations of cephalosporin, ciprofloxacin, and imipenem. eGNR, enteric gram-negative rods. 
Table 3. Comparison of clinical characteristics of MDR versus non-MDR and ESBL vs. non-ESBL producers among gram-negative Enterobacteriaceae

\begin{tabular}{|c|c|c|c|c|c|c|}
\hline Variable & $\begin{array}{l}\text { Non-MDR } \\
(n=186)\end{array}$ & $\begin{array}{c}\text { MDR } \\
(n=143)\end{array}$ & $P$ value & $\begin{array}{l}\text { Non-ESBL producing } \\
(\mathrm{n}=275)\end{array}$ & $\begin{array}{c}\text { ESBL producing } \\
(n=54)\end{array}$ & $P$ value \\
\hline Male sex & $116(62.4)$ & $76(53.2)$ & 0.117 & 158 & 34 & 0.549 \\
\hline Age (yr) & $4.3(2.7-8.0)$ & $5.6(3.7-13.1)$ & 0.003 & $4.9(2.9-9.3)$ & $5.5(3.1-30.3)$ & 0.093 \\
\hline Fever day at admission (day) & $2(1-3)$ & $2(1-4)$ & 0.022 & $2(1-3)$ & $2(1-4)$ & 0.75 \\
\hline Total fever duration (day) & $3(2-4)$ & $3(2-4)$ & 0.118 & $3(2-4)$ & $3(2-5)$ & 0.904 \\
\hline Time to defervescence (day) & $1(0-2)$ & $1(0-2)$ & 0.732 & $1(0-2)$ & $1(0-2)$ & 0.965 \\
\hline Duration of hospitalization (day) & $6(5-7)$ & $6(5-7)$ & 0.012 & $6(5-7)$ & $6(5-8)$ & 0.015 \\
\hline Total WBC count $\left(\times 10^{3} / \mu \mathrm{L}^{3}\right)$ & $14.5(10.6-18.7)$ & $15.6(12.5-19.7)$ & 0.103 & $15.4(11.7-19.1)$ & $15.0(11.1-19.4)$ & 0.676 \\
\hline C-reactive protein (mg/dL) & $4.2(2.2-7.9)$ & $4.8(2.8-8.3)$ & 0.315 & $4.4(2.3-8.3)$ & $4.7(1.5-7.7)$ & 0.844 \\
\hline
\end{tabular}

Values are presented as number (\%) or median (interquartile range).

MDR, multidrug-resistant; ESBL, extended-spectrum beta-lactamase; WBC, white blood cell.

Boldface indicates a statistically significant difference with $P<0.05$.

Table 4. Comparison of recurrence rates depending on the presence of VUR in patients with fUTI caused by ESBL vs. nonESBL producers

\begin{tabular}{|c|c|c|c|}
\hline Variable & $\begin{array}{c}\text { ESBL } \\
\text { producer }\end{array}$ & $\begin{array}{l}\text { Non-ESBL } \\
\text { producer }\end{array}$ & $P$ value \\
\hline Patients without VUR & $3 / 46(6.5)$ & $12 / 255(4.7)$ & 0.602 \\
\hline \multicolumn{4}{|l|}{ Patient with VUR ${ }^{a)}$} \\
\hline Total recurrence & $6 / 8(75.0)$ & $9 / 20(45.0)$ & 0.15 \\
\hline Recurrence within 6 months & $6 / 8(75.0)$ & $6 / 20(30.0)$ & 0.03 \\
\hline
\end{tabular}

VUR, vesicoureteral reflux; fUTI, febrile urinary tract infection; ESBL, extended-spectrum beta-lactamase.

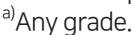

Boldface indicates a statistically significant difference with $P<0.05$.

$30 \%$. The susceptibility rates to cephalosporins including extended-spectrum cephalosporins (3rd and 4th generation) also decreased by greater than $10 \%$ during this period. The rapidly declining susceptibility rates, owing to the selective pressure caused by the overuse of penicillins and cephalosporins, is a major problem. A systematic review investigating the prevalence of antibiotic resistance in urinary tract infections caused by $E$. coli in children showed that children who received antibiotics in primary care were more likely to have bacteria from the urinary tract resistant to antibiotics, persisting up to 6 months. ${ }^{14)}$ Children are overexposed to these classes of antibiotics in primary care, therefore, continual regulation and discretion in prescribing these classes of antibiotics are essential.

Decreased susceptibility to ciprofloxacin was observed after 2010. Recent studies in adults have demonstrated increasing resistance against fluoroquinolone, especially in eGNRs, because fluoroquinolone is one of the most commonly prescribed antimicrobials. ${ }^{16,17)}$ The resistance gene against fluoroquinolone can be acquired from mother's microbiota, and even antibiotic naïve patients who live in high-level of fluoroquinolone-resistant community could show the same susceptibility pattern ${ }^{18)}$. Other possibilities may include the association between fluoroquinolone resistance and ESBL-production. According to multivariate analysis, the percentages of resistance to ciprofloxacin in the ESBL-positive group was greater than negative group. ${ }^{19,20)}$

Piperacillin-tazobactam, on the other hand, did not show any decrease in the susceptibility rate throughout the 11-year period
(Fig. 2), and did not show an increasing trend in resistance (Fig. 4). An intervention study carried out at a children's hospital showed that substituting piperacillin-tazobactam in place of extended-spectrum cephalosporins successfully decreased the prevalence of infections caused by ESBL-producing K. pneumoniae and E. coli. ${ }^{21)}$ Therefore, with the high susceptibility rate and hardly any change in resistance, piperacillin-tazobactam may be a good choice as the first-line antibiotics for fUTI instead of the commonly used extended-spectrum cephalosporins for the reduction of ESBL-producing Enterobacteriaceae. ${ }^{22)}$

Even with fUTI caused by ESBL-producing eGNRs increasing at an alarming rate, many studies have demonstrated that noncarbapenem antibiotics were as effective as administering carbapenems for the treatment of community-onset fUTI caused by ESBL-producing eGNRs in both adults and children. ${ }^{23,24)}$ Treatment failure or relapse rates between patients who were administered a noncarbapenem for ESBL-producing eGNRs did not differ compared to those administered a carbapenem. ${ }^{23,24)}$ In this study, patients without VUR also showed similar results, with no treatment failure and low short-term recurrences. However, when further investigating patients with underlying VUR, patients who had fUTI caused by an ESBL producer had a significantly higher short-term recurrence rate than those with non-ESBL-producing eGNRs, regardless of the fact that adequate treatment was given and all showed complete clinical improvement. Because this was a univariate analysis and the number of patients with VUR was small, further studies on patients with VUR are needed to confirm whether this patient group will benefit from using a carbapenem rather than a noncarbapenem for fUTI caused by ESBL-producing eGNR.

The worldwide emergence of carbapenem-resistant Enterobacteriaceae (CRE) has become a threat to public health, and recently, there have been reports of increasing prevalence of carbapenemase-producing CRE in both nosocomial and community settings. ${ }^{25-27)}$ Because all the study participants in this study had no underlying diseases and no previous history of fUTI, carbapenemase-producing CRE or resistance to aminoglycoside was not an issue. However, during 2016, 2 noncarbapenemase-producing CRE emerged as pathogens of fUTI 
in children without recent admission histories. The emergence of CREs warrants continuous monitoring of antibiotic resistance in children.

There were several limitations to this study. First, because this was a single center study, caution is necessary in applying the results for nationwide purposes. However, the advantage that this study had in being a single center study was that the same medical team followed the same protocol for evaluation and treatment of the patients included in this study. Therefore, interpersonal bias may be reduced. Also, much of the clinical outcome parameters were univariate analyses. Further multicenter studies with prospective designs are needed for multivariate analyses in order to remove of confounding factors.

In conclusion, the epidemiologic patterns of fUTI showed that the disease prevalence was highest below 12 months of age and female predominance from 12 months old and above. The percentage of fUTI caused by MDR and ESBL-producing eGNRs is increasing at a rapid rate, and antibiotic resistance to not only the commonly prescribed primary care antibiotics such as ampicillin-sulbactam and cephalosporins, but also to ciprofloxacin and imipenem in increasing with a linear upward trend, which warrants careful monitoring of antibiotic usage and resistance. Piperacillin-tazobactam may be an adequate first-choice antibiotic for combating resistance. Finally, using carbapenem can be considered for the patients with underlying VUR who have fUTI caused by an ESBL-producing eGNR to reduce short-term recurrence rate. Further multicenter studies are needed to confirm these findings.

\section{Conflicts of interest}

No potential conflict of interest relevant to this article was reported.

See the commentary "Should we prescribe carbapenem for treating febrile urinary tract infection caused by extendedspectrum $\beta$-lactamase-producing Enterobacteriaceae in children with vesicoureteral reflux?" via https://doi.org/10.3345/cep. 2020.01830 .

\section{References}

1. Marild S, Jodal U. Incidence rate of first-time symptomatic urinary tract infection in children under 6 years of age. Acta Paediatr 1998;87:549-52.

2. Hellstrom A, Hanson E, Hansson S, Hjalmas K, Jodal U. Association between urinary symptoms at 7 years old and previous urinary tract infection. Arch Dis Child 1991;66:232-4.

3. Shaikh N, Morone NE, Bost JE, Farrell MH. Prevalence of urinary tract infection in childhood: a meta-analysis. Pediatr Infect Dis J 2008;27:3028.

4. Jakobsson B, Svensson L. Transient pyelonephritic changes on 99mTechnetium-dimercaptosuccinic acid scan for at least five months after infection. Acta Paediatr 1997;86:803-7.

5. Coulthard MG, Lambert HJ, Vernon SJ, Hunter EW, Keir MJ, Matthews JN. Does prompt treatment of urinary tract infection in preschool children prevent renal scarring: mixed retrospective and prospective audits. Arch Dis Child 2014;99:342-7.
6. Finnell SM, Carroll AE, Downs SM, Subcommittee on urinary tract I. Technical report-diagnosis and management of an initial UTI in febrile infants and young children. Pediatrics 2011;128:e749-70.

7. Stein R, Dogan HS, Hoebeke P, Kocvara R, Nijman RJ, Radmayr C, et al. Urinary tract infections in children: EAU/ESPU guidelines. Eur Urol 2015;67:546-58.

8. Subcommittee on Urinary Tract Infection SCoQI, Management, Roberts $\mathrm{KB}$. Urinary tract infection: clinical practice guideline for the diagnosis and management of the initial UTI in febrile infants and children 2 to 24 months. Pediatrics 2011;128:595-610.

9. Montini G, Toffolo A, Zucchetta P, Dall'Amico R, Gobber D, Calderan A, et al. Antibiotic treatment for pyelonephritis in children: multicentre randomised controlled non-inferiority trial. BMJ 2007;335:386.

10. Performance standards for antimicrobial susceptibility testing; twentyfifth informational supplement. Wayne (PA): Clinical Laboratory Standards Institute, 2015.

11. Magiorakos AP, Srinivasan A, Carey RB, Carmeli Y, Falagas ME, Giske CG, et al. Multidrug-resistant, extensively drug-resistant and pandrugresistant bacteria: an international expert proposal for interim standard definitions for acquired resistance. Clin Microbiol Infect 2012;18:26881.

12. Lee KY. New insights for febrile urinary tract infection (acute pyelonephritis) in children. Child Kidney Dis 2016:37-44.

13. Huh SM, Park BK, Kang HM, Rhim JW, Suh JS, Lee KY. Clinical implications of DMSA scan in childhood acute pyelonephritis. Child Kidney Dis 2017;21:107-13.

14. Bryce A, Hay AD, Lane IF, Thornton HV, Wootton M, Costelloe C. Global prevalence of antibiotic resistance in paediatric urinary tract infections caused by Escherichia coli and association with routine use of antibiotics in primary care: systematic review and meta-analysis. BMJ 2016;352:1939.

15. Lee DS, Choe HS, Lee SJ, Bae WJ, Cho HJ, Yoon BI, et al. Antimicrobial susceptibility pattern and epidemiology of female urinary tract infections in South Korea, 2010-2011. Antimicrob Agents Chemother 2013;57:5384-93.

16. Saksena R, Nayyar C, Manchanda V. Six-year susceptibility trends and effect of revised Clinical Laboratory Standards Institute breakpoints on ciprofloxacin susceptibility reporting in typhoidal Salmonellae in a tertiary care paediatric hospital in Northern India. Indian J Med Microbiol 2016;34:520-5.

17. Kim YA, Park YS, Youk T, Lee H, Lee K. Trends in South Korean antimicrobial use and association with changes in Escherichia coli resistance rates: 12-year ecological study using a nationwide surveillance and antimicrobial prescription database. PLoS One 2018;13:e0209580.

18. Saksena R, Gaind R, Sinha A, Kothari C, Chellani H, Deb M. High prevalence of fluoroquinolone resistance amongst commensal flora of antibiotic naïve neonates: a study from India. J Med Microbiol 2018;67:481-8.

19. Azap OK, Arslan H, Serefhanoğlu K, Colakoğlu S, Erdoğan H, Timurkaynak F, et al. Risk factors for extended-spectrum beta-lactamase positivity in uropathogenic Escherichia coli isolated from communityacquired urinary tract infections. Clin Microbiol Infect 2010;16:147-51.

20. Pitout JD, Nordmann P, Laupland KB, Poirel L. Emergence of Enterobacteriaceae producing extended-spectrum beta-lactamases (ESBLs) in the community. J Antimicrob Chemother 2005;56:52-9.

21. Lee J, Pai H, Kim YK, Kim NH, Eun BW, Kang HJ, et al. Control of extended-spectrum beta-lactamase-producing Escherichia coli and Klebsiella pneumoniae in a children's hospital by changing antimicrobial agent usage policy. J Antimicrob Chemother 2007;60:629-37.

22. Bantar C, Vesco E, Heft C, Salamone F, Krayeski M, Gomez H, et al. Replacement of broad-spectrum cephalosporins by piperacillin-tazobactam: impact on sustained high rates of bacterial resistance. Antimicrob Agents Chemother 2004;48:392-5.

23. Abe Y, Inan-Erdogan I, Fukuchi K, Wakabayashi H, Ogawa Y, Hibino S, et al. Efficacy of non-carbapenem antibiotics for pediatric patients with first febrile urinary tract infection due to extended-spectrum beta-lactamaseproducing Escherichia coli. J Infect Chemother 2017;23:517-22. 
24. Lee B, Kang SY, Kang HM, Yang NR, Kang HG, Ha IS, et al. Outcome of antimicrobial therapy of pediatric urinary tract infections caused by extended-spectrum beta-lactamase-producing Enterobacteriaceae. Infect Chemother 2013;45:415-21.

25. Xu Y, Gu B, Huang M, Liu H, Xu T, Xia W, et al. Epidemiology of carbapenem resistant Enterobacteriaceae (CRE) during 2000-2012 in Asia. J Thorac Dis 2015;7:376-85.

26. Khatri A, Naeger Murphy N, Wiest P, Osborn M, Garber K, Hecker $\mathrm{M}$, et al. Community-acquired pyelonephritis in pregnancy caused by KPC-producing Klebsiella pneumoniae. Antimicrob Agents Chemother 2015;59:4375-8.
27. Logan LK, Weinstein RA. The epidemiology of carbapenem-resistant Enterobacteriaceae: the impact and evolution of a global menace. J Infect Dis 2017;215:S28-36.

How to cite this article: Suh W, Kim BN, Kang HM, Yang EA, Rhim JW, Lee KY. Febrile urinary tract infection in children: changes in epidemiology, etiology, and antibiotic resistance patterns over a decade. Clin Exp Pediatr 2021;64:293-300. https://doi.org/10.3345/cep.2020.00773 